DOE/ER/15291-2

\title{
CONFORMATIONAL PROPERTIES AND ENTROPIC PARTITIONING OF TOPOLOGICALLY COMPLEX POLYMERS UNDER CONFINEMENT
}

\section{FINAL REPORT}

For Period April 1, 2002 through December 31, 2004

\author{
Fernando A. Escobedo
}

Cornell University

Ithaca, New York 14853

March 2005

Prepared for

\section{THE U.S. DEPARTMENT OF ENERGY}

AWARD NO. DE-FG02-02ER15291

\section{NOTICE}

This report was prepared as an account of work sponsored by the United States Government. Neither the United States nor the United States Department of Energy, nor any of their employees, nor any of their contractors, subcontractors, or their employees, makes any warranty, express or implied, or assume any legal liability or responsability for the accuracy, completeness, or usefulness of any information, apparatus, product or process disclosed or represents that its use would not infringe privately-owned rights. 


\section{AWARD NO. DE-FG02-02ER15291}

\section{A. Development of Human Resources and Outreach.}

Funding from this award was primarily employed for the support of a graduate student Zhong Chen, who defended his Ph.D. Thesis in January of 2004, graduated from Cornell (May 2005) and currently holds a post-doctoral position at the University of Georgia, Atlanta. Some support was also provided for Jianfang Zhong who unfortunately failed her qualifying exam and left the department in December 2004 with a terminal Masters of Engineering degree. Results from this work have been disseminated both in journal publications (see below), meetings (AIChE annual meetings), seminars in several universities, and the "Polymer Outreach Program" for industry organized by Cornell University.

\section{B. Publications.}

Total support from this grant is acknowledged in the following papers:

1. Z. Chen and F. Escobedo, "Influence of Polymer Architecture and Polymer-wall Interaction on Partitioning of Polymers into a Slit", Phys. Rev. E, 69, 021802 (2004).

2. Z. Chen and F. Escobedo, "Simulation of Chain-length Partitioning in a Microfabricated Channel via Entropic Trapping", Molecular Simulation 29, 417 (2003).

Partial support from this grant is acknowledged in the following papers:

3. F. Escobedo and Z. Chen, "Liquid crystalline behavior of a semifluorinated oligomer", J. Chem. Phys. 121, 11463 (2004).

4. C. Abreu, J. Zhong, and F. Escobedo, "Rebridging Configuration-Bias Method for Regular Lattices. Application to Multi-Cyclic Polymers", draft to be submitted to Macromolecules.

The first three papers are also being submitted as post-prints via the DOE E-link system.

\section{Major Scientific Findings and Accomplishments Background}

The main objective of this project was to study the connection between molecular topology and structure of chain molecules in confined environments via molecular simulation. At the core of this problem lies the quantification of molecular entropy as a key driving force that determines the "shape" and "mobility" of the molecules. In fact, entropy measures the ability of a chain to adopt different conformations (e.g., in the folded and unfolded states of proteins), to move around different regions of a system, and to pack and assemble into larger structures. When chains are confined in small pores, for example, their "shape" and mobility will change in a way that is not readily predictable, especially for chains with complex architecture. This phenomenon is relevant to numerous applications of polymers such as in chromatographic separations, electrophoresis, colloidal stabilization, thin-film processing, and the preparation of clay-based nanocomposites. For example, the so-called high osmotic pressure chromatography is intended for large-scale fractionation of polydisperse polymer solutions. Entropy-driven surface segregation of certain components in a confined polymer solution or melt could also be used to functionalize the surface of thin films. 
Summary of Results on the effect of chain architecture on the properties of confined polymers The effect of molecular topology (e.g., branch and loop structures) on the solution properties of polymers is subtle and not well characterized. Because the conformational entropy of a polymer depends on its topology, many properties are affected by it such as its size and shape, mobility, bulk-to-pore partitioning, adsorption strength on surfaces, and depletion-induced forces on colloidal surfaces. We have systematically studied the effect of molecular topology on the structure and entropic partitioning of linear, branched, hyper-branched, cyclic, and hyper-cyclic polymers in a bulk solution and in pores. Ours is the first simulation study aimed at characterizing the conformational properties of hyper-cyclic molecules.

Key findings: Our results show how differences in molecular architecture can be used to partition polymers in a porous media e.g., a highly branched polymer tends to be depleted in narrow pores (smaller than the coil size) relative to a less branched chain of equal molecular weight, but this trend is reversed in wide pores. It was also found that intra-molecular crosslinking (associated with cyclic structures) is an effective way to tune the conformational entropy of a polymer; the more crosslinks a molecule has, the smaller its conformational entropy, and the easier it is to adsorb it onto attractive pore walls. Intra-crosslinked chains are thus more effective steric stabilizer of colloid particles than linear chains (which are better depleting agents).

Simulations were also used to investigate the mechanism of entropic trapping for model linear and branched DNA molecules as they go from a deep channel to a shallow channel driven by an electric field. In such a system, a molecule whose radius of gyration is larger than the gap of the shallow channel tends to get temporarily trapped at its entrance. Our results show that at moderate and strong fields, longer chains escape faster than shorter ones because, in the absence of significant differences in the free energy barrier for escape, larger chains access a larger entrance area to the narrow channel; these results are in agreement with reported experimental observations.

These and other accomplishments are discussed in detail below.

1. Chain adsorption in slit pores [Publication \#1: Phys. Rev. E, 69, 021802 (2004)]. We investigated the effects of molecular topology and polymer-surface interaction on the properties of isolated polymer chains trapped in an attractive slit via off-lattice Monte Carlo simulations. Such studies are important since different "regimes" of liquid chromatography for polymers in good solvent can be reproduced in a unified framework by varying the polymerwall adsorption energy $\varepsilon$. Novel methods were implemented to allow efficient simulation of molecular structures, confinement forces, and free energies for chains interacting with such "sticky" surfaces. Six different chain architectures were studied (see Fig. 1): linear, starbranched, dendritic, cyclic, two-node (i.e., containing two tetrafunctional intramolecular crosslinks), and six-node molecules. The first three topologies entail increasing degree of branching, and the last three topologies entail increasing degree of intramolecular bonding. The confinement force and conformational properties for all these systems in a good solvent were compared (for identical molecular weight "N"). The focus was on the compensation point where the wall attraction counterbalances the polymer-slit exclusion effects; at this point the 
confinement force " $F$ " vanishes. It was found that the attractive energy at the compensation point " $\mathrm{E}$ " is a weak increasing function of the chain length (smaller $\mathrm{E}$ is associated with betteradsorbing molecules). Due to their globular shape and numerous chain ends, stars and dendrimers experience a relatively small entropic penalty for adsorption at low adsorption force and moderate confinement. However, as the adsorption force increases, linear chains have a smaller E. In moderate to weak confinement, molecules with intramolecular crosslinks, such as cyclic and multi-node molecules, always adsorb better than the other chains (with the same N, see Fig. 2). At strong confinement conditions, chain rigidity becomes the dominating factor and the more flexible linear chain adsorbs the best at all adsorption strengths.

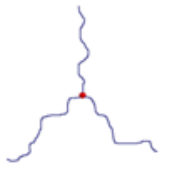

Star

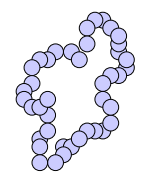

Cyclic

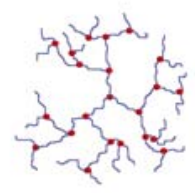

Dendrimer

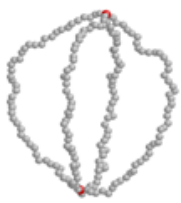

Multicyclic: Two Nodes

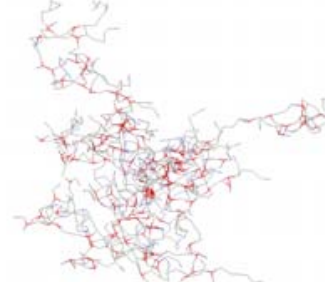

Hyperbranched

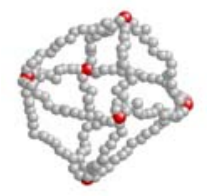

Hypercyclic: Six Nodes

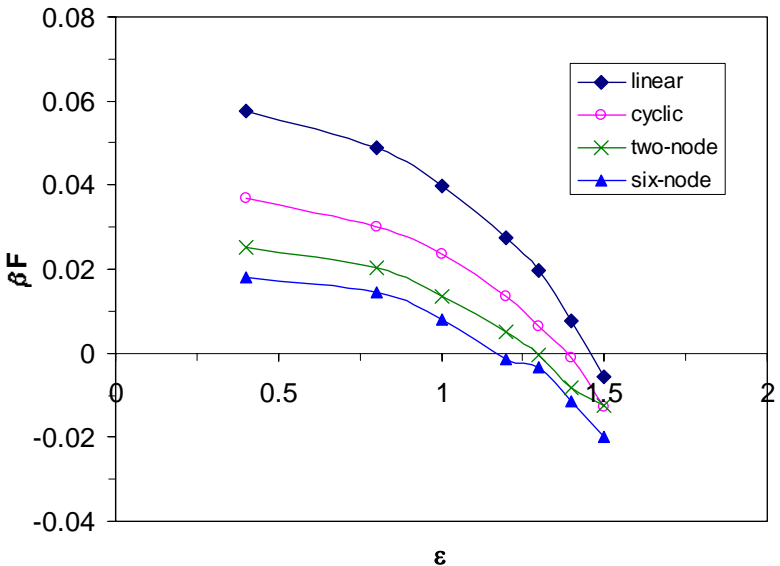

Figure 2: Confinement force $\beta \mathrm{F}$ as a function of adsorption strength $\varepsilon$ for linear, cyclic, two-node, and sixnode molecules with molecular weight $N=186$ in a slit with a wall separation of $D=32$. The compensation point, $\varepsilon=\mathrm{E}$, occurs when $\beta \mathrm{F}=0$.

Confinement forces can also be related to depletion forces in dilute-polymer colloid mixtures if colloidal particles are larger than the polymer coils so that their surfaces can be approximated by planar walls. Because $\mathrm{F}$ relates to the osmotic pressure difference between the bulk solution and the confined zones, a positive $\mathrm{F}$ indicates that polymers will tend to be excluded from the gap (of separation D) between two colloidal particles and "push" the particles together (depletion attraction). On the contrary, a negative $\mathrm{F}$ indicates a polymer-mediated repulsion between colloidal particles that provides steric stabilization. Besides the polymer-wall interaction strength $\varepsilon_{2}$ our results show that polymer topology could also be used to control $\mathrm{F}$ and thus colloidal phase behavior. For equal N, linear chains should be more efficient depleting agents (larger positive $\mathrm{F}$ values for non- or weak-adsorption conditions), while intracrosslinked molecules should be more effective stabilizers (smaller, more negative $\mathrm{F}$ values at a given $\varepsilon$, and a lower threshold $\mathrm{E}$ for steric stabilization).

2. Chain partitioning via entropic barriers [Publication \#2: Molec. Simul. 29, 417 (2003)]

A molecular simulation approach was used to investigate the mechanism of entropic trapping for model linear DNA molecules as they go from a deep channel to a shallow channel driven 
by an electric field (Fig. 3). This is a model system whose understanding could guide experimentalist in the design and optimization of novel micro-fabricated devices for efficient separations. In such a system, a molecule whose radius of gyration is larger than the gap of the shallow channel will tend to get temporarily trapped at its entrance.

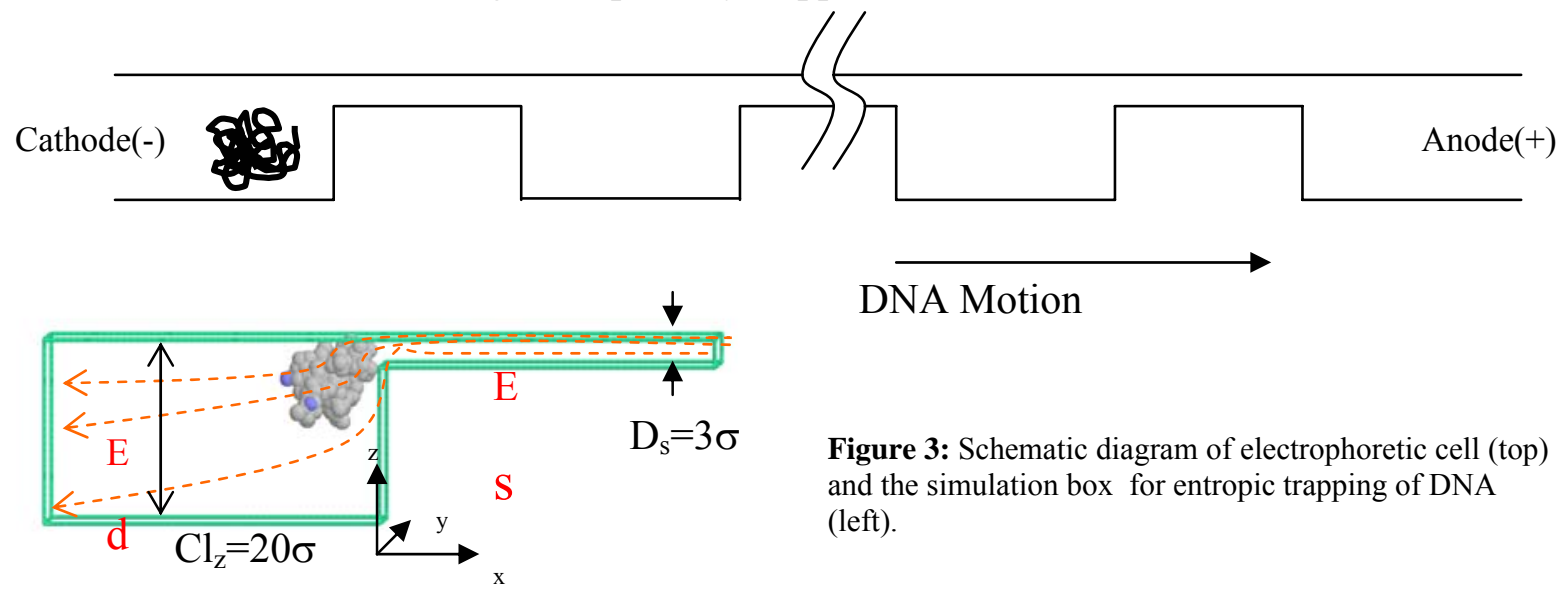

The simulations probed the thermodynamics (free energy of the molecules as a function of chain position) and the kinetics (trapping times) of entropic trapping. Most of our results refer to chains with lengths ranging from $\mathrm{N}=40$ to $\mathrm{N}=320$ (unperturbed radii of gyration $R_{g}{ }^{0}$ range from $2.6 \sigma$ to $7.4 \sigma$ where $\sigma$ is the bond length=Kuhn segment), which correspond to DNA fragments from $10 \mathrm{kbp}$ to $100 \mathrm{kbp}$. It was fount that in weak and moderate electrical fields, the free energy barrier for escape $\Delta F$ increases with chain length approaching a plateau value; in a strong electrical field, $\Delta F$ exhibits a mild decreasing trend with chain length. As shown in Fig. 4-left, at weak electric fields, shorter chains escape faster than longer chains because of their lower associated $\Delta F$. At moderate and strong fields, longer chains escape faster than shorter ones because, in the absence of significant differences in $\Delta F$, larger chains access a larger entrance area to the narrow channel; these results are in agreement with reported experimental observations. Results for model branched chains (stars) suggest that coil diameter is the determinant factor of escape rates (Fig. 4-right).
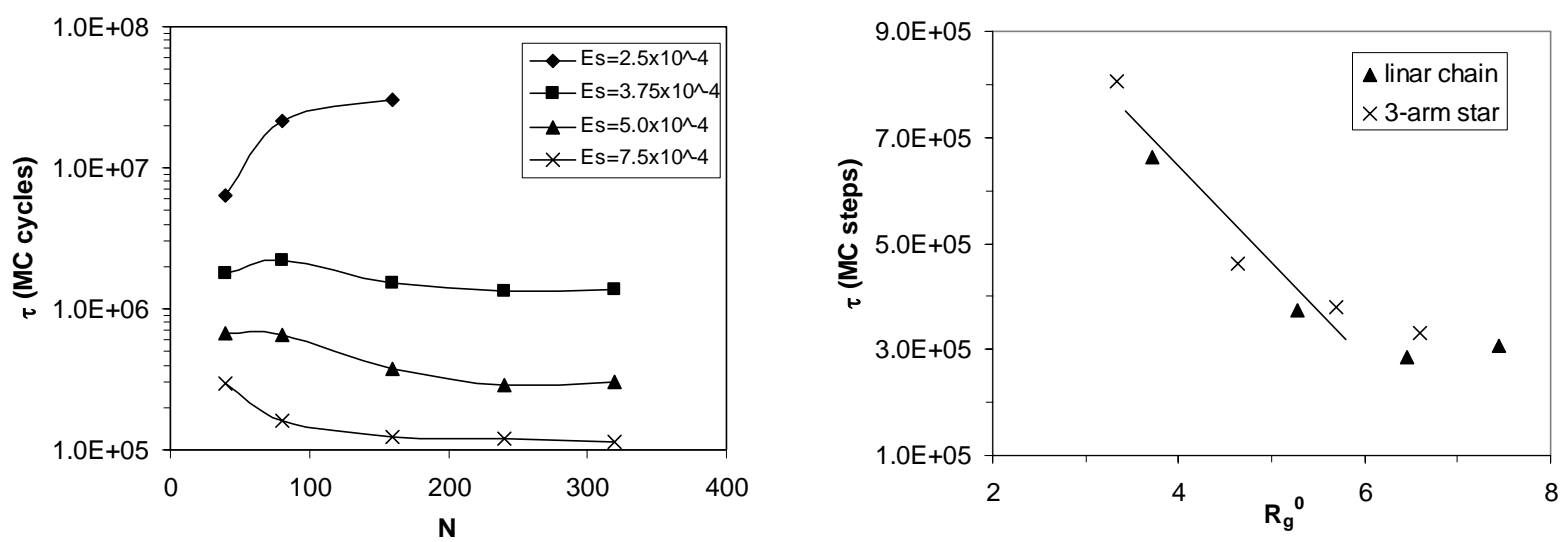

Figure 4: Left: Trapping time as a function of chain length at different fields (Es=dimensionless electrical field reduced by $k_{B} T / e \sigma$, where $e$ is the electron charge). Right: Results for Linear vs. branched at Es $=5 \times 10^{4}$. 
This work is still ongoing. Dr. Zhong Chen (funded for nearly 2 years by this grant while a graduate student) has yet not finish another paper wherein we implemented a more realistic Brownian Dynamics method to simulate kinetic entropic trapping for a wider range of branched and cyclic DNA chains. This work is expected to be completed during 2005 but this is contingent of Dr. Chen finding enough "free" time in his new postdoctoral position.

\section{Scaling and coil-to-globule transitions in multi-cyclic chains (draft)}

In this work (partially supported by this grant) we examine how the size of a molecule (e.g., its radius of gyration "Rg") and its coil-to-globule transition temperature scale as a function of molecular weight $(\mathrm{N})$ for the types of molecules similar to those shown in Fig. 1. Especial attention is placed on multi-cyclic chains since they constitute a novel class of polymers. Our results show that, compared to linear chains, such multi-cyclic chains are significantly more compact though obey a similar scaling exponent in $\operatorname{Rg} \sim \mathrm{N}^{v}$. Also, the coil-to-globule transition in multi-cyclic chains is much more diffuse compared to the "sharp" transition temperature observed in linear chains. These results can be explained by the reduced conformational entropy of multi-cyclic chains, which in turn reduces the differences between the coil and globule states. This work has not yet been submitted for publication.

\section{Effect of entropic mismatch on block copolymer mesophases [Publication \#3: J. Chem.}

Phys. 121, 11463 (2004)].

The interplay between chain conformation and energetic interactions in multi-chain systems was also investigated by simulating the self-assembly of model diblock copolymers into smectic layers. This work (only partially supported by this grant) is a departure from previous studies in that it is concerned with multi-chain (melt) systems where intermolecular "energetic" interactions are also strong. The diblock copolymer investigated has an alkyl block and a perfluoro-alkyl block which leads to both energetic and entropic mismatches and to a rich phase behavior. By lowering the temperature the system transitions from a disordered melt to several quasi-ordered "mesophases". The energetic mismatch between the blocks initially drives such a transition via microphase segregation toward a lamellar-like phase. However, mismatches in both the stiffness and thickness of the blocks lead to an "entropic" driving force toward efficient packing of space that frustrates the conventional lamellar structure. As a result, the system "finds" alternative novel microsegregated structures, a "checkerboard" phase and a bilayer phase (both smectic phases). Our results, and extensions thereof, could potentially be useful to guide the design of smart chain architectures that exploit entropic mismatch to produce novel temperature-induced phase transitions. Such transitions, in turn, could find applications in sensors, actuators, and mimics of muscle action.

Results from this work partially served as the basis for the formulation of the new proposal that this PI submitted to DOE in September 2004 (submission \# ER04-01-11734-14029-1490), titled: "Simulation of Complex Microphase Formation in Pure and Nanoparticle-filled Diblock Copolymers". 